\title{
Determinación de bajas concentraciones de hidrógeno en aleaciones de circonio utilizando radiografía de neutrones como técnica no destructiva
}

\section{Determination of low concentrations of hydrogen in zirconium alloys using neutron radiography as a non-destructive technique}

\author{
Nayibe Lucia Buitrago ${ }^{1,2,4}$, Javier Santisteban ${ }^{1,2,3,4}$, Julio Marín ${ }^{3,4}$, \\ Aureliano Tartaglione ${ }^{1,3,5}$, Mark Daymond ${ }^{6}$, Mirco Grosse ${ }^{7}$
}

\footnotetext{
${ }^{1}$ CONICET, Godoy Cruz 2290 (C1425FQB), Buenos Aires, Buenos Aires, Argentina.

${ }^{2}$ Instituto Balseiro, Av. Exequiel Bustillo 9500, San Carlos de Bariloche, Bariloche, Río Negro, Argentina.

${ }^{3}$ CNEA, Av. Del Libertador 8250 (C1429BNP), Buenos Aires, Buenos Aires, Argentina.

${ }^{4}$ Centro Atómico Bariloche, Av. Exequiel Bustillo 9500, San Carlos de Bariloche, Bariloche, Río Negro, Argentina.

${ }^{5}$ Centro Atómico Constituyentes, Av. Gral. Paz 1499, B1650 Villa Maipú, Buenos Aires, Buenos Aires, Argentina.

${ }^{6}$ Department of Mechanical and Materials Engineering, Queen's University, Kingston, Canada K7L 3N6, Kingston, Ontário,Canada.

${ }^{7}$ Karlsruhe Institute of Technology, Karlsruhe, Baden-Württemberg, Alemania.

e-mail: buitrago@cab.cnea.gov.ar
}

\section{RESUMEN}

Las aleaciones base circonio ( $\mathrm{Zr}$ ) se utilizan en la fabricación de las vainas de combustible y en componentes estructurales de los reactores de potencia por su baja absorción neutrónica. Estas aleaciones son susceptibles al daño por hidrógeno $(\mathrm{H})$, debido a que la concentración del mismo puede incrementarse localmente en respuesta a gradientes de temperaturas o tensiones. Esto provoca una degradación general de sus propiedades mecánicas, y puede generar un proceso lento de degradación conocido como rotura diferida inducida por hidruros (Delayed Hydride Cracking - DHC). El contenido de $\mathrm{H}$ en aleaciones de circonio es generalmente determinado mediante técnicas destructivas. En este trabajo determinamos el contenido de $\mathrm{H}$ en el rango 0300 wt ppm con una sensibilidad de $\sim 5$ wt ppm y una resolución espacial de $\sim 0.1 \mathrm{~mm}$ utilizando la radiografía de neutrones (neutrografía) como técnica no destructiva. A fin de comparar la capacidad de distintas facilidades de neutrografía para este tipo de análisis, los estudios se realizaron en cuatro laboratorios con características diferentes en términos del flujo y de la distribución en energía de los neutrones incidentes sobre la muestra, así como la máxima resolución espacial disponible en cada caso (parámetro L/D). Las facilidades fueron ANTARES en el reactor FRM-II (Alemania), Engin-X en la fuente pulsada ISIS (Reino Unido), BOA en la fuente de espalación SINQ (Suiza), y la facilidad de neutrografía del reactor RA-6 de Bariloche (Argentina). La resolución espacial alcanzada permite determinar el coeficiente de difusión de $\mathrm{H}$ en aleaciones de $\mathrm{Zr}$ utilizando muestras de dimensiones aproximadas de $5 \times 10 \times 10 \mathrm{~mm}^{3}$.

Palabras clave: Radiografía de neutrones, Circonio, Hidrógeno, DHC.

\begin{abstract}
Zircon base alloys $(\mathrm{Zr})$ are used in the manufacture of fuel sheaths and structural components of power reactors because of their low neutron absorption. These alloys are susceptible to damage by hydrogen $(\mathrm{H})$ because the concentration of the alloys can be increased locally in response to temperature or stress gradients. This causes a general degradation of its mechanical properties, and can generate a degradation process known as Delayed Hydride Cracking (DHC). H content in zirconium alloys is generally determined by destructive techniques. In this work we determine the $\mathrm{H}$ content in the range 0-300 wt ppm with a sensitivity of $\sim 5 \mathrm{wt}$ ppm and a spatial resolution of $\sim 0.1 \mathrm{~mm}$ using neutron radiography as a nondestructive technique. In order to compare the capacity of different neutrography facilities for this type of analysis, the studies were carried out
\end{abstract}


in four laboratories with different characteristics in terms of the flow and the energy distribution of the incident neutrons on the sample, as well as the maximum spatial resolution available in each case (L/D parameter). The facilities were ANTARES in the FRM-II reactor (Germany), Engin-X in the pulsed source ISIS (UK), BOA in the SINQ (Switzerland) sputtering source, and the neutron facility at the Bariloche RA-6 reactor (Argentina). The spatial resolution obtained allows to determine the diffusion coefficient of $\mathrm{H}$ in $\mathrm{Zr}$-alloys using samples of $5 \times 10 \times 10 \mathrm{~mm}^{3}$, approximately.

Keywords: Neutron radiography, Zirconium, Hydrogen, DHC.

\section{INTRODUCCIÓN}

Las aleaciones de $\mathrm{Zr}$ son ampliamente utilizadas en componentes de reactores nucleares, principalmente en las vainas de los elementos combustibles y en los tubos de presión (reactores CANDU y RBMK). Estos reactores refrigerados con agua, operan a alta temperatura $\left(280^{\circ} \mathrm{C}-350^{\circ} \mathrm{C}\right)$ y alta presión $\sim 90 \mathrm{~atm}$, estas condiciones facilitan que parte del hidrógeno $(\mathrm{H})$ presente en el agua ingrese en las aleaciones de $\mathrm{Zr}$ ubicándose como átomos intersticiales, de forma que a medida que aumenta la concentración de $\mathrm{H}$ tiende a precipitar formando hidruros. Además, el $\mathrm{H}$ migra hacia regiones con alta concentración de tensiones, y al exceder la solubilidad termina precipitando allí en forma de hidruros. Estos hidruros fragilizan el material alcanzando la fractura a tensiones menores que las de diseño.

A fin de evitar el deterioro de las propiedades mecánicas, el contenido de $\mathrm{H}$ presente en los componentes base $\mathrm{Zr}$ de reactores nucleares está fijado por normas. Por ejemplo, los tubos de presión de $\mathrm{Zr} 2.5 \% \mathrm{Nb}$ deben tener un máximo de 15 wt ppm $\mathrm{H}$ previo a su instalación. La determinación de $\mathrm{H}$ se realiza habitualmente por métodos destructivos como la fusión en gas inerte, la extracción en vacío [1,2], etc. En este trabajo comparamos el rendimiento de diferentes facilidades de neutrografía para la determinación de bajos contenidos de $\mathrm{H}$ en forma no destructiva.

El coeficiente de difusión de $\mathrm{H}$ en aleaciones de $\mathrm{Zr}$ se ha determinado tradicionalmente por mediciones de concentración de $\mathrm{H}$ en juntas de difusión de dimensiones de decenas de $\mathrm{cm}$, a partir de pequeñas muestras seccionadas de las mismas tras tiempos largos de difusión, obteniendo diferentes valores para distintas direcciones de un mismo componente [1,2]. Nuestro objetivo final apunta a utilizar la técnica de neutrografía para la determinación del coeficiente de difusión de $\mathrm{H}$ en tubos de presión de aleaciones base $\mathrm{Zr}$ de $\sim 5 \mathrm{~mm}$ de pared para temperaturas cercanas a las de operación $\left(\sim 300{ }^{\circ} \mathrm{C}\right)$. Esto requiere la utilización de muestras de dimensiones aproximadas de 10x10x $5 \mathrm{~mm}^{3}$, conteniendo perfiles de concentración de $\mathrm{H}$ entre 0 y $150 \mathrm{wt}$ ppm. En este trabajo se pretende determinar perfiles con una sensibilidad de $\sim 5 \mathrm{wt}$ ppm $\mathrm{H}$ y resolución espacial de $\sim 0.1 \mathrm{~mm}$.

A fin de comparar el rendimiento de las distintas facilidades de neutrografía, en este trabajo inicialmente se realizaron experimentos sobre un conjunto de muestras homogéneas con concentraciones de $\mathrm{H}$ conocidas en un laboratorio de la Argentina y tres laboratorios internacionales (Alemania, Suiza y Reino Unido), a fin de estimar la sensibilidad, la resolución espacial y los tiempos de medición involucrados en cada caso. Seguidamente, se realizaron experimentos sobre una muestra conteniendo un perfil de concentración de $\mathrm{H}$ en dos de las facilidades relevadas. Presentamos a continuación una breve descripción de las muestras utilizadas, las facilidades experimentales empleadas y los resultados de los experimentos realizados.

\section{MATERIALES}

Todas las muestras fueron producidas por el Departamento de Ingeniería Mecánica y de Materiales, de la Universidad Queens en Canadá. Las muestras utilizadas se dividen en dos grupos, 1) 14 muestras de calibración, homogéneas, para determinar la sensibilidad al H, 2) 1 muestra con perfil de concentración, inhomogénea, para determinar la resolución espacial obtenible. Las muestras fueron cortadas de placas de $\mathrm{Zr} 2.5 \mathrm{Nb}$ y Zircaloy-2, las cuales tenían la microestructura resultante del proceso de fabricación para los tubos de presión utilizados en los reactores CANDU.

Se utilizaron doce muestras homogéneas de calibración de Zircaloy-2 y dos de $\mathrm{Zr} 2.5 \% \mathrm{Nb}$. Todas las muestras tenían originalmente dimensiones de $10 \times 10 \times 4 \mathrm{~mm}^{3}$. Tras medirlas en dos de las facilidades, se cortó una sección de $2.5 \times 10 \times 4 \mathrm{~mm}^{3}$ de las mismas para determinar su contenido de $\mathrm{H}$ en forma destructiva por extracción caliente en vacío según norma (ASTM E 146-83), obteniendo los resultados listados en la Tabla 1. El procedimiento de preparación de estas muestras consistió en la incorporación de $\mathrm{H}$ por carga catódica (ánodos de $\mathrm{Pb}, 24$ horas a $80{ }^{\circ} \mathrm{C}$ ), seguido de una posterior homogenización por recocido a $350{ }^{\circ} \mathrm{C}$ durante 24 horas. Debido a la casi nula solubilidad del $\mathrm{H}$ en $\mathrm{Zr}$ a temperatura ambiente, en todos los experimentos realizados el $\mathrm{H}$ se encuentra disperso en las muestras en forma de precipitados micrométricos de hidruro de $\mathrm{Zr}$. 
La muestra con perfil de concentración, de dimensiones $10 \times 10 \times 4 \mathrm{~mm}^{3}$, fue cortada de una placa de $\mathrm{Zr} 2.5 \mathrm{Nb}$ laminada en caliente a $860{ }^{\circ} \mathrm{C}$. La muestra fue pulida en todas sus caras para que se formara una capa hidruro homogénea durante la carga catódica en solución acuosa de ácido sulfúrico $\left(200 \mathrm{ml}_{2} \mathrm{O}+5 \mathrm{ml}\right.$ $\mathrm{H}_{2} \mathrm{SO}_{4}$ ) a $85{ }^{\circ} \mathrm{C}$ durante 30 horas. A continuación la muestra fue pulida nuevamente, pero sólo en 5 de sus caras, de manera que una cara conserve la capa de hidruro. La muestra fue posteriormente recocida en aire a $350{ }^{\circ} \mathrm{C}$ durante 90 minutos a fin de provocar la difusión de $\mathrm{H}$ desde la capa de hidruro hacia el interior del metal. La muestra fue retirada del horno y enfriada en aire hasta temperatura ambiente a fin de "congelar" el perfil de difusión así generado. Como resultado, el $\mathrm{H}$ aparece también aquí en forma de precipitados, pero con una mayor concentración de los mismos en las zonas cercanas a la capa de hidruro. La Figura 1 muestra un esquema de la muestra identificando la capa de hidruro y sus dimensiones.

Tabla 1: Contenido de $\mathrm{H}$ de las muestras de calibración.

\begin{tabular}{|c|c|c|c|c|c|c|c|c|c|c|c|c|c|c|}
\hline MUESTRA & 1 & 2 & 3 & 4 & 5 & 6 & 7 & 8 & 9 & 10 & 11 & 12 & 13 & 14 \\
\hline MATERIAL & $\mathrm{Zr} 2.5 \mathrm{Nb}$ & $\mathrm{Zr} 2$ & $\mathrm{Zr} 2$ & $\mathrm{Zr} 2$ & $\mathrm{Zr} 2$ & $\mathrm{Zr} 2$ & $\mathrm{Zr} 2$ & $\mathrm{Zr} 2$ & $\mathrm{Zr} 2.5 \mathrm{Nb}$ & $\mathrm{Zr} 2$ & $\mathrm{Zr} 2$ & $\mathrm{Zr} 2$ & $\mathrm{Zr} 2$ & $\mathrm{Zr} 2$ \\
\hline ESPESOR $( \pm 0.1 \mathrm{~mm})$ & 7.3 & 7.3 & 7.4 & 7.2 & 7.4 & 7.6 & 7.3 & 7.5 & 7.3 & 7.4 & 7.4 & 7.4 & 7.1 & 7.3 \\
\hline$[\mathrm{H}]( \pm 5$ wt ppm) & 7 & 11 & 14 & 26 & 37 & 58 & 64 & 84 & 130 & 166 & 171 & 175 & 248 & 366 \\
\hline
\end{tabular}

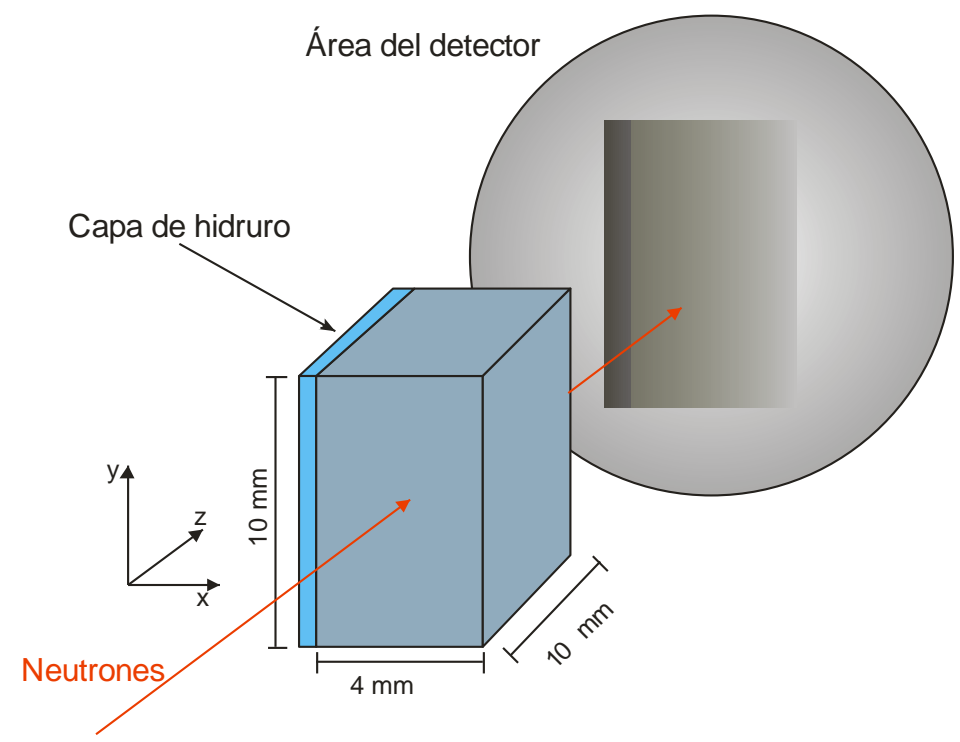

Figura 1: Geometría de las muestras inhomogéneas y esquema del experimento.

\section{MÉTODOS}

\subsection{Radiografía con neutrones (Neutrografía)}

La Figura 1 muestra un esquema del experimento de neutrografía realizado sobre una muestra, en este caso la muestra con perfil de concentración. Un haz colimado de neutrones de intensidad $I_{0}$ incide sobre la misma, y la intensidad $I_{S}$ del haz transmitido es registrada por un detector. La transmisión de la muestra es

$$
\operatorname{Tr}=\frac{I_{s}-B}{I_{0}-B}
$$

donde $B$ es el fondo, es decir, la señal registrada por el detector cuando el haz de neutrones se encuentra cerrado. La transmisión depende del espesor z y del coeficiente de atenuación $\sum$ de la muestra,

$$
\operatorname{Tr}=\exp (-z * \Sigma)
$$

Así, en experimentos con muestras de espesor uniforme como las que utilizamos aquí, la variación de la transmisión registrada por el detector se debe principalmente a cambios en el coeficiente de atenuación, en 
nuestro caso particular debido a variaciones en el contenido de $\mathrm{H}$ de las muestras. La Ec. (2) permite estimar la sensibilidad que debe poseer la medición para poder observar los contenidos de H de interés. Así, el cambio de intensidad $\Delta I_{H}$ registrado en el detector para una muestra base $\mathrm{Zr}$ que contiene una fracción de volumen de hidruro $c_{H}$ es:

$$
\frac{\Delta I_{H}}{I_{Z r}}=\frac{I_{Z r}-I_{Z r+H}}{I_{Z r}}=c_{H} z\left(\Sigma_{H}-\Sigma_{Z r}\right)
$$

El coeficiente de atenuación de las aleaciones base $\mathrm{Zr}$ para neutrones térmicos es $\sim 0.24 \mathrm{~cm}^{-1}$, en tanto el del hidruro de $\mathrm{Zr}$ es aproximadamente veinte veces mayor, $\sim 4.8 \mathrm{~cm}^{-1}$. Un contenido de $\mathrm{H}$ de $5 \mathrm{wt}$ ppm corresponde a una fracción de volumen de hidruro de $\sim 0.0003$, por lo que para nuestras muestras de $1 \mathrm{~cm}$ de espesor estimamos que las mediciones deben tener una sensibilidad del orden de $\Delta I_{H} / I_{Z r} \sim 0.001$ en el número de cuentas transmitidas por la muestra. Si se considera una estadística de Poisson, donde la incerteza relativa de una señal en la que se registraron $N$ cuentas es $1 / \sqrt{N}$, obtenemos que necesitamos registrar un mínimo de $\sim 10^{6}$ cuentas para cada muestra analizada. El rango dinámico y la sensibilidad a eventos espurios de los sistemas de detección utilizados no permiten registrar este número de cuentas en una sola medición. Por esto, en todos los casos se tomó un cierto número de imágenes $N_{i}$, y se procesó estadísticamente este conjunto de imágenes a fin de conseguir la desviación estándar requerida. Para los sistemas basados en una placa centelladora acoplada a una cámara CCD se calculó la mediana de cada una de las imágenes registradas $\left(\tilde{I}_{S}, \tilde{I}_{0}, \tilde{B}_{)}\right.$, ya que esta resulta menos sensible a los llamados "puntos blancos", eventos esporádicos que saturan el valor de pixeles aislados. A partir de las mismas se calculó la imagen transmisión mediana $\widetilde{T r}$ de acuerdo a la Ec. (1). En todos los casos se realizó además un posterior promediado espacial de la transmisión sobre un número de pixeles que pertenecieran a una zona de interés bien definida, en la que no se esperase una variación significativa de la señal registrada.

Como el valor de la transmisión depende del espesor de la muestra investigada, la calibración y los resultados se presentan en términos del coeficiente de atenuación del material, que se calcula a partir de las magnitudes experimentales de acuerdo a:

$$
\Sigma=\frac{-\ln \left(\frac{\tilde{I}_{s}-\tilde{B}}{\tilde{I}_{0}-\tilde{B}}\right)}{z}
$$

\subsection{Instrumentos utilizados}

Las estaciones de radiografía de neutrones en las que se realizaron los experimentos sobre las muestra homogéneas son: (i) ANTARES [3,4] del reactor FRM-II en Alemania, (ii) BOA [5] de la fuente SINQ en Suiza, (iii) Engin-X [6] de la fuente Isis en el Reino Unido, y (iv) el instrumento de neutrografía del reactor RA-6 [7] en Argentina. Estas facilidades tienen características diferentes en cuanto a la forma de producción y la intensidad de fuente de neutrones, el material moderador de donde emergen los neutrones, el tamaño $D$ de la fuente efectiva de neutrones (diámetro del pinhole), la distancia $L$ a la que el detector se encuentra del mismo, el flujo incidente sobre la muestra, y sistema de detección (material sensible a neutrones, resolución espacial). La Tabla 2 lista las características de las facilidades utilizadas en este trabajo. Engin-X es un difractómetro diseñado para la medición de tensiones internas que se encuentra al final de una guía de neutrones curva, y no está optimizado para la adquisición de imágenes. Sin embargo, es posible configurarlo para este fin mediante un detector provisto temporariamente por el Departamento de Ciencias del Espacio de la Universidad de California en Berkeley [8].

Las muestras fueron ubicadas delante del haz mediante dispositivos fabricados ad hoc, a fin de maximizar el número de especímenes que podían ser investigados en forma simultánea. La Figura 2(a) muestra el arreglo utilizado en el instrumento ANTARES, en el que se midieron dos muestras por cada imagen registrada. El número de imágenes adquiridas, los tiempos de exposición para cada imagen y las cuentas totales registradas en cada caso también fueron variables. Los detalles para cada facilidad se presentan en la Tabla 3.

Los experimentos sobre la muestra con perfil de concentración se realizaron sólo en las facilidades Engin-X y ANTARES. En este caso, la cuantificación del contenido de H se realizó a partir de los experimentos realizados sobre las muestras homogéneas. 


\section{RESULTADOS}

\subsection{Muestras homogéneas}

Los valores de los coeficientes de atenuación medidos para cada una de las muestras homogéneas en las cuatro facilidades visitadas se muestran en la Figura 3. En todos los casos los valores presentan una dependencia lineal con el contenido de $\mathrm{H}$ medido en las mismas en forma destructiva. Se observa que los puntos correspondientes a las muestras de Zircaloy-2 se encuentran sobre una recta, en tanto las dos muestras de $\mathrm{Zr} 2.5 \% \mathrm{Nb}$ se hallan sistemáticamente por encima de dicha recta. Así, hemos realizado ajustes lineales de cuadrados mínimos sólo sobre los valores correspondientes a muestras de Zircaloy 2, obteniendo las rectas que se muestran en la figura. La Tabla 4 lista los valores de la pendiente y la ordenada al origen ajustados en cada caso, junto a la desviación estándar de los puntos alrededor de cada recta.

Tabla 2: Principales características de las facilidades utilizadas.

\begin{tabular}{|c|c|c|c|c|c|c|c|c|}
\hline FACILIDAD & FUENTE & ESPECTRO & $\begin{array}{l}\text { MODERADOR Y } \\
\text { TEMPERATURA }\end{array}$ & $\begin{array}{l}\text { LAMBDA } \\
\text { MEDIO (Å) }\end{array}$ & $\begin{array}{c}\text { FLUJO } \\
\left(\mathrm{N} \mathrm{cm}^{-2} \mathrm{~s}^{-1}\right)\end{array}$ & L/D & DETECTOR & $\begin{array}{l}\text { TAMAÑO DE } \\
\text { PIXEL }(\mathrm{mm})\end{array}$ \\
\hline ANTARES & $\begin{array}{l}\text { Reactor } \\
\text { FRM-II }\end{array}$ & Frio & $\mathrm{D}_{2}-18 \mathrm{~K}$ & 1.8 & $1 \times 10^{8}$ & 510 & $\begin{array}{c}\text { Pantalla de } \\
\text { centelleo } \\
\text { Cámara } \\
\text { CCD }\end{array}$ & 0.02 \\
\hline Engin- $X$ & $\begin{array}{l}\text { Fuente de } \\
\text { espalación }\end{array}$ & Frio & $\mathrm{CH}_{4}-110 \mathrm{~K}$ & 2 & $5 \times 10^{6}$ & & $\begin{array}{c}\text { Microplacas } \\
\text { dopadas con } \\
\text { boro }\end{array}$ & 0.055 \\
\hline BOA & $\begin{array}{l}\text { Fuente de } \\
\text { espalación }\end{array}$ & Frio & $\mathrm{D}_{2}-25 \mathrm{~K}$ & 1.5 & $1 \times 10^{8}$ & N/A & $\begin{array}{c}\text { Pantalla de } \\
\text { centelleo } \\
\text { Cámara } \\
\text { CCD } \\
\end{array}$ & 0.013 \\
\hline RA-6 & $\begin{array}{l}\text { Reactor } \\
\text { RA-6 }\end{array}$ & Térmico & $\mathrm{H}_{2} \mathrm{O}-293 \mathrm{~K}$ & $\sim 1.1$ & $6.6 \times 10^{7}$ & 82 & $\begin{array}{l}\text { Pantalla de } \\
\text { centelleo } \\
\text { Cámara } \\
\text { CCD }\end{array}$ & 0.168 \\
\hline
\end{tabular}
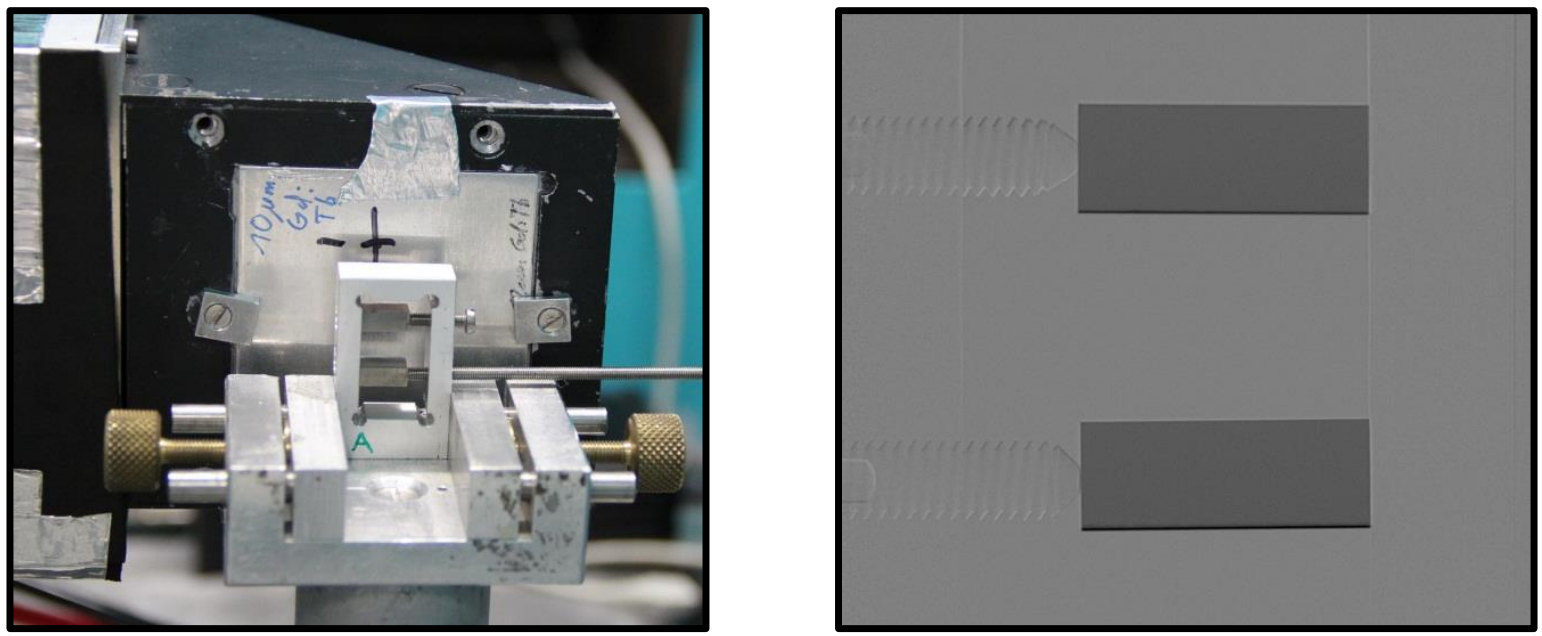

Figura 2: (a) Arreglo experimental utilizado en la facilidad ANTARES del reactor FRM-II, Munich, Alemania (b) Imagen de la transmisión obtenida. 
Tabla 3: Características del protocolo de medición utilizado.

\begin{tabular}{c|c|c|c}
\hline & $\begin{array}{c}\text { TIEMPO DE } \\
\text { ADQUISICIÓN } \\
(\mathbf{s})\end{array}$ & $\begin{array}{c}\text { NÚMERO DE } \\
\text { IMÁGENES } \boldsymbol{N}_{\boldsymbol{i}}\end{array}$ & $\begin{array}{c}\text { CUENTAS } \\
\text { PROMEDIO } \\
\text { POR PIXEL }\end{array}$ \\
\hline ANTARES & 10 & 40 & 626 \\
\hline Engin-X & 900 & 1 & 35000 \\
\hline BOA & 180 & 1 & 12000 \\
\hline RA-6 & 20 & 50 & 29000 \\
\hline
\end{tabular}

El valor absoluto de la ordenada en el origen varía entre $0.16 \mathrm{~cm}^{-1}$ y $0.24 \mathrm{~cm}^{-1}$ para las distintas facilidades, valor determinado principalmente por la distribución energética del espectro de neutrones incidente sobre la muestra. Las pendientes de las rectas varían entre $\sim 0.00009$ y $\sim 0.00022\left(\mathrm{~cm}^{-1} / \mathrm{wt} \mathrm{ppm} \mathrm{H}\right)$. La pendiente da una medida de la sensibilidad de cada facilidad al contenido de $\mathrm{H}$ presente en la muestra. Las facilidades que presentan la mayor sensibilidad son ANTARES y Engin-X, con pendientes de $\sim 0.00022\left(\mathrm{~cm}^{-1} / \mathrm{wt}\right.$ ppm H), en tanto la facilidad del reactor RA-6 presenta un valor $\sim 50 \%$ menor. Cabe destacar que la sensibilidad de esta técnica para resolver el contenido de $\mathrm{H}$ depende fuertemente del espesor de la muestra analizada, según se desprende de la Ec. (3).

Tabla 4: Parámetros de ajuste de cada recta de calibración.

\begin{tabular}{c|c|c|c|c}
\hline FACILIDAD & $\begin{array}{c}\text { PENDIENTE }\left(\boldsymbol{S}_{H}\right) \\
\left(\mathbf{c m}^{-1} / \mathbf{w t} \mathbf{~ p p m ~ H )}\right.\end{array}$ & $\begin{array}{c}\text { ORDENADA AL } \\
\text { ORIGEN }\left(\mathbf{c m}^{-1}\right)\end{array}$ & $\begin{array}{c}\text { DESVIACIÓN } \\
\text { ESTÁNDAR }(\xi) \\
\left(\mathbf{c m}^{-1}\right)\end{array}$ & $\begin{array}{c}\text { SENSIBILIDAD } \\
\boldsymbol{\delta}_{\boldsymbol{H}}(\mathbf{w t} \mathbf{~ p p m ~ H )}\end{array}$ \\
\hline ANTARES & $(2.23 \pm 0.02) 10^{-4}$ & $0.2208 \pm 0.0003$ & 0.0015 & 6 \\
\hline Engin-X & $(2.24 \pm 0.01) 10^{-4}$ & $0.2349 \pm 0.0001$ & 0.0022 & 9 \\
\hline BOA & $(1.91 \pm 0.01) 10^{-4}$ & $0.1714 \pm 0.0002$ & 0.0032 & 16 \\
\hline RA-6 & $(0.92 \pm 0.01) 10^{-4}$ & $0.1566 \pm 0.0002$ & 0.0014 & 15 \\
\hline
\end{tabular}

Para realizar una comparación cuantitativa de la exactitud esperada para el valor del contenido de $\mathrm{H}$ resultante de las mediciones realizadas en cada equipo, definimos la exactitud esperable de la medición como $\delta_{H}=\xi / S_{H}$, con $\xi$ la desviación cuadrática de los puntos experimentales alrededor de la recta de ajuste, y $S_{H}$ la pendiente de la recta. La Tabla 4 lista los valores de exactitud esperada así calculados, los cuales se encuentran en el rango de 6-16 wt ppm $\mathrm{H}$.

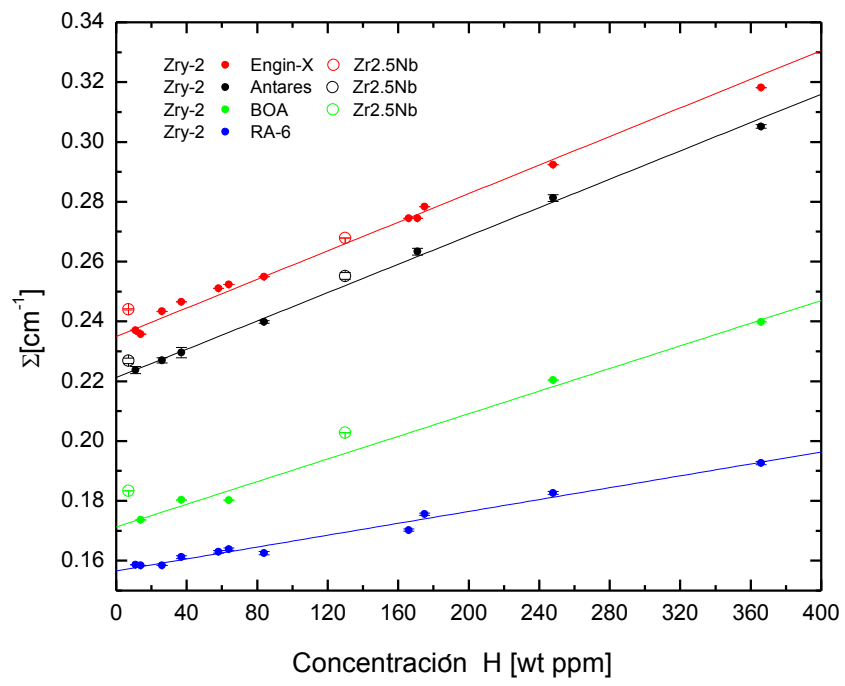


Figura 3: Rectas de calibración, en función de la concentración de hidrógeno y el coeficiente de atenuación, para cuatro facilidades de radiografía de neutrones.

\subsection{Muestra con perfil de concentración}

La Figura 4 muestra los perfiles de concentración de $\mathrm{H}$ medidos en la muestra inhomogénea de $\mathrm{Zr} 2.5 \% \mathrm{Nb}$, en función de la distancia a la capa de hidruro. La primera medición se realizó en Engin-X, y con la experiencia adquirida se optimizó el arreglo experimental y la estrategia de adquisición de imágenes de los experimentos realizados posteriormente en ANTARES. Así, los perfiles medidos en ANTARES presentan mejor estadística y resolución espacial. Los errores asignados a cada curva corresponden a la precisión estimada en la sección anterior. Los valores de contenido de $\mathrm{H}$ fueron calculados a partir del coeficiente de atenuación medido utilizando las rectas de calibración presentadas en la Figura 3, pero desplazadas verticalmente a fin de que pasen por los puntos correspondientes a las muestras de $\mathrm{Zr} 2.5 \% \mathrm{Nb}$. Los valores de coeficiente de atenuación fueron medidos sobre regiones de $23 \mathrm{~mm}^{2}$ en Engin-X y sobre $27 \mathrm{~mm}^{2}$ en ANTARES.

En esta muestra la capa de hidruro actúa como fuente de $\mathrm{H}$, por lo que los perfiles de concentración medidos pueden describirse analíticamente por la ecuación de difusión de un soluto en un medio semiinfinito [2]:

$$
C\left(x, t_{\text {recocido }}\right)=\left(C_{T}-C_{0}\right) \operatorname{erfc}\left(\frac{x}{2 \sqrt{D t_{\text {recocido }}}}\right)+C_{0}
$$

donde $x$ es la distancia a la capa de hidruro, $t_{\text {recocido }}$ es el tiempo de recocido, $C_{0}$ es la concentración de $\mathrm{H}$ lejos de la capa de hidruro antes del recocido, $C_{T}$ es la solubilidad de $\mathrm{H}$ en $\mathrm{Zr}$ a la temperatura de recocido, y $D$ coeficiente de difusión de $\mathrm{H}$ en la aleación. La Figura 4 muestra los ajustes de cuadrados mínimos realizados fijando el valor de $C_{T}$ a $126.3 \mathrm{wt}$ ppm $\mathrm{H}$, (valor reportado para la solubilidad de $\mathrm{H}$ a $350{ }^{\circ} \mathrm{C}$ [9]) y dejando como parámetros de ajuste $D$ y $C_{0}$. Los valores de coeficiente de difusión obtenidos para esta muestra de $\mathrm{Zr} 2.5 \% \mathrm{Nb}$ son $(2.7 \pm 0.5) \times 10^{-10} \mathrm{~m}^{2} / \mathrm{s}$ y $(2.0 \pm 0.7) \times 10^{-10} \mathrm{~m}^{2} / \mathrm{s}$ para las mediciones ANTARES y Engin-X, respectivamente.

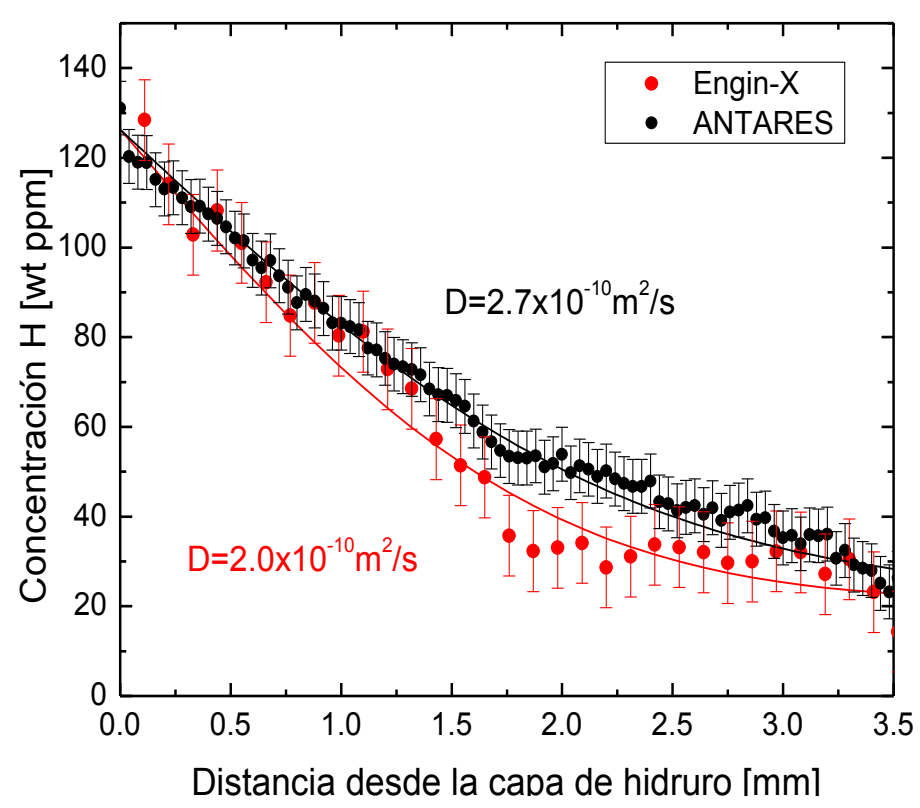

Figura 4: Perfiles de difusión medidos en dos facilidades (Engin-X y ANTARES) para la muestra con una capa de hidruro, tras someterla a un recocido de 90 minutos a $350^{\circ} \mathrm{C}$, que provoca la difusión de $\mathrm{H}$ hacia el interior.

\section{DISCUSIÓN}

En la Figura 3 hemos presentado las rectas de calibración obtenidas para las cuatro facilidades visitadas. Los resultados muestran que en todas ellas es posible determinar contenidos de $\mathrm{H}$ en el rango 0-400 wt ppm $\mathrm{H}$ con una exactitud de $\sim 6-16$ wt ppm $\mathrm{H}$, sobre muestras que presenten una sección transversal al haz de neutrones en el rango de las decenas de $\mathrm{mm}^{2}$, y un espesor en el rango de los $\mathrm{mm}$. En tanto se mantengan los 
mismos espectros incidentes y sistemas de detección, las rectas ajustadas pueden ser directamente utilizadas como calibración de la técnica para la medición de especímenes de Zircaloy 2 con contenidos de $\mathrm{H}$ desconocidos.

Para estimar la exactitud de estas mediciones se evaluó la desviación estándar de los puntos experimentales alrededor de la recta ajustada, que en todos casos fue considerablemente mayor que la incerteza estadística involucrada en la medición del coeficiente de atenuación. Esto indica errores sistemáticos debido a variaciones en el coeficiente de atenuación que presenta cada muestra, por razones distintas al contenido de $\mathrm{H}$ presente en las mismas. Para identificar las causas de estas variaciones, la Figura 5(b) muestra la dependencia del coeficiente de atenuación con la longitud de onda de los neutrones incidentes para el hidruro de circonio, y para las dos aleaciones utilizadas en distintas condiciones metalúrgicas. Estas son un Zircaloy 2 con una distribución isotrópica de orientaciones microcristalinas (como se observaría en un polvo muy compactado), y un tubo de presión de $\mathrm{Zr} 2.5 \% \mathrm{Nb}$. En este último caso, se presenta el coeficiente de atenuación a lo largo de dos de sus direcciones principales (radial y axial). El valor del coeficiente de atenuación determinado experimentalmente corresponde al promedio del coeficiente de atenuación sobre el rango de longitudes de onda utilizadas, pesado por el espectro incidente $\phi(\lambda)$ y la eficiencia de detección $\varepsilon(\lambda)$, ambos también dependientes de la longitud de onda $\lambda$. A modo de ejemplo, la Figura 5 (a) muestra el peso efectivo $I_{0}^{*}(\lambda)=$ $\phi(\lambda) \varepsilon(\lambda)$ correspondiente al instrumento Engin-X [6]. Observamos que para las longitudes de onda de interés, el coeficiente de atenuación de las aleaciones base $\mathrm{Zr}$ presenta variaciones con la orientación que pueden dar origen a pequeñas variaciones en el coeficiente de atenuación promedio medido en cada caso. Las muestras de calibración homogéneas de Zircaloy 2 de 10x10x4 $\mathrm{mm}^{3}$ fueron producidas a partir de una chapa laminada que presentaba textura cristalográfica, por lo que es de esperar que existan pequeñas variaciones en el coeficiente de atenuación, según como se oriente la muestra en el haz de neutrones. Durante las mediciones no se prestó atención a este detalle, y los experimentos se realizaron orientando indistintamente cualquiera de los dos lados de $10 \mathrm{~mm}$ a lo largo de la dirección del haz.

En combinación con los datos presentados en la Tabla 2, la Figura 5 también provee una interpretación para los valores de ordenada al origen y de pendiente listados en la Tabla 4. La pendiente de las rectas de calibración es proporcional al valor del coeficiente de atenuación del hidruro para longitudes de onda cercanas a la longitud de onda media reportada para cada facilidad. Por otro lado, el valor de la ordenada al origen dependerá del peso efectivo del coeficiente de atenuación que se observa a longitudes de onda más largas que el promedio espectral.

La variación que observamos entre el coeficiente de atenuación medido para las muestras de Zircaloy 2 y Zr2.5\% Nb parece originarse en efectos de la microestructura, ya que la variación de la composición química entre estas aleaciones posee un impacto menor sobre esta magnitud. 


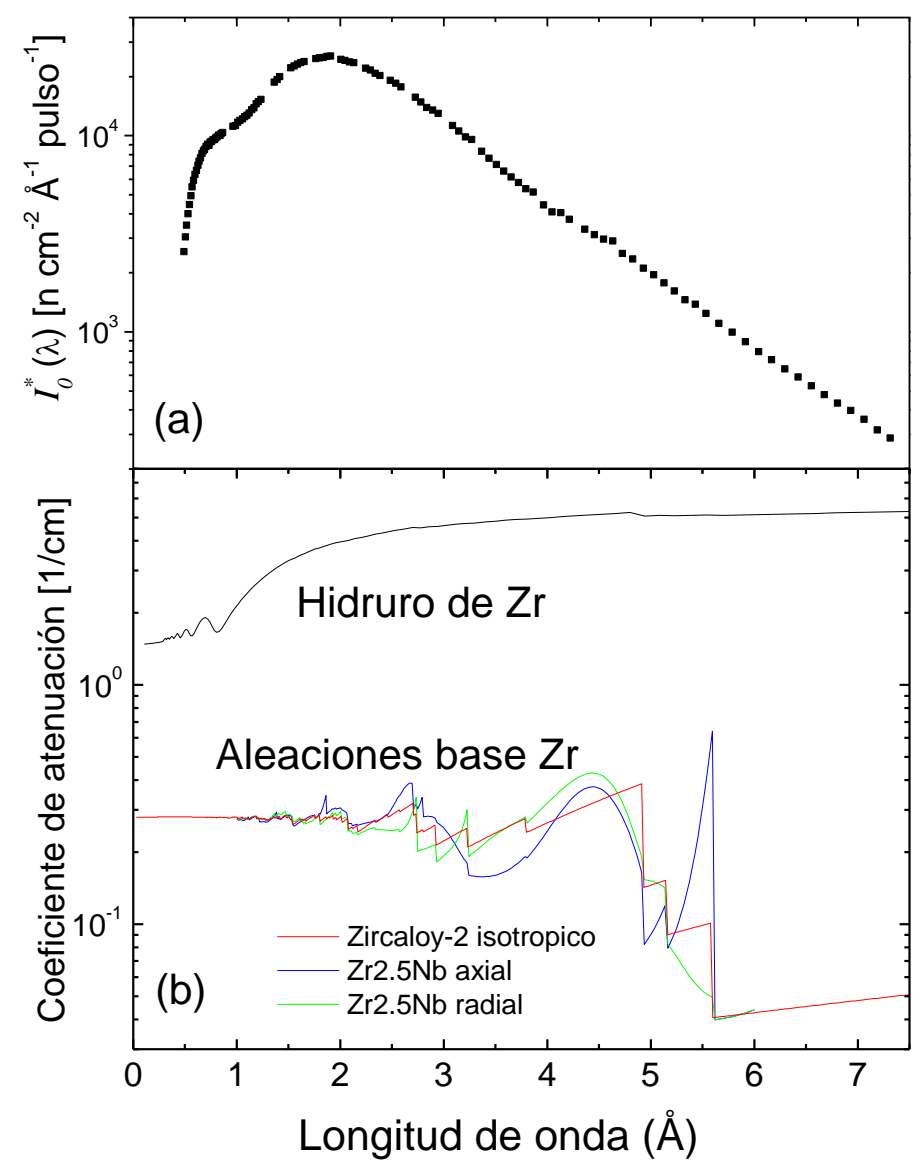

Figura 5: (a) Espectro de neutrones determinado por el detector para el instrumento Engin-X. (b) Coeficiente de atenuación en aleaciones base Zr y en hidruro de Zirconio en función de la longitud de onda.

La resolución espacial para la determinación de $\mathrm{H}$ es otro parámetro importante, ya que nos indica la mínima distancia que se puede diferenciar en la imagen. Este valor se estimó multiplicando el número de pixeles agrupados (determinado por el protocolo de medición adoptado - ver sección 3.1) por el tamaño de un pixel. La mejor resolución espacial se consiguió en las mediciones realizadas en la facilidad ANTARES $(\sim 80 \mu \mathrm{m})$, mientras que en los experimentos en Engin-X se obtuvo una resolución de $\sim 200 \mu \mathrm{m}$. Cabe destacar que este tipo de experimentos podrían realizarse en BOA, pero no en el instrumento de RA-6, ya que requieren de una resolución espacial que no está disponible en dicho equipo. Se espera que estos experimentos puedan ser realizados en el tomógrafo de neutrones que será instalado en el reactor RA-10.

El coeficiente de difusión de hidrógeno en aleaciones de $\mathrm{Zr}$ ha sido obtenido utilizando métodos destructivos [2]. Para un tratamiento de difusión a $300{ }^{\circ} \mathrm{C}$ los cálculos teóricos arrojan un valor de $D$ de $5.7 \mathrm{e}^{-}$ ${ }^{11} \mathrm{~m}^{2} / \mathrm{s}$, mientras que los valores medidos son diferentes para cada dirección de difusión: $5.7 \mathrm{e}^{-11} \mathrm{~m}^{2} / \mathrm{s}$ para dirección radial y $7.2 \mathrm{e}^{-11} \mathrm{~m}^{2} / \mathrm{s}$ para la dirección transversal. De manera que $D$ cambia dependiendo la dirección de difusión debido a los cambios en la microestructura que presentan las láminas, creados durante el proceso de fabricación. En nuestro estudio los valores de $D$ obtenidos $\left(2.7 \times 10^{-10}\right.$ en ANTARES y $2.0 \times 10^{-10}$ en Engin$\mathrm{X})$ resultan mayores al coeficiente de difusión teórico a $350{ }^{\circ} \mathrm{C}\left(D_{t 350}=1.2 \mathrm{e}^{-10} \mathrm{~m}^{2} / \mathrm{s}\right)$. Teniendo en cuenta la variación de $D$ con la microestructura se plantea realizar mediciones para las diferentes direcciones de difusión a fin de determinar el intervalo de $D$ para la temperatura mencionada.

\section{CONCLUSIONES}

La técnica de radiografía de neutrones permite determinar el contenido de $\mathrm{H}$ en aleaciones base $\mathrm{Zr}$ en forma no destructiva, en muestras de dimensiones en el rango de milímetros a centímetros, para concentraciones de $\mathrm{H}$ en el rango 0-400 wt ppm $\mathrm{H}$, con una exactitud de $\sim 6-16$ wt ppm $\mathrm{H}$ y con una resolución espacial de hasta $\sim 80 \mu \mathrm{m}$.

Para la realización de este tipo de análisis es importante definir un protocolo de medición que permita alcanzar la estadística necesaria optimizando las capacidades experimentales disponibles en cada facilidad. 
Bajo estas condiciones, es posible determinar coeficientes de difusión de $\mathrm{H}$ en $\mathrm{Zr}$ en muestra con dimensiones menores a $10 \mathrm{~mm}$.

\section{AGRADECIMIENTOS}

Este trabajo se realizó con el financiamiento otorgado por el CONICET (PIP-11220110100542) y por el Organismo Internacional de Energía Atómica (IAEA Research Contract 17252).

\section{BIBLIOGRAFÍA}

[1] KHATAMIAN, D., "Deuterium diffusion along the three principal directions in anisotropic $\mathrm{Zr}-2.5 \mathrm{Nb}$ ", Journal of Alloys and Compounds. v. 580, pp. s58-s62, Mar 2013.

[2] CRANK, J., "In: The Mathematics Diffusion", second ed. s.l. : Oxford University Press, pp. 32-47, 1975.

[3] TREMSIN, A. S., MÜHLBAUER, M. J., SCHILLINGER, B., et al., "High resolution stroboscopic neutron radiography at the FRM-II ANTARES facility", IEEE Trans. Nucl. Sci. v. 57, pp. 2955-2962, Oct 2010.

[4] SANTISTEBAN, J. R., VICENTE-ALVAREZ, M. A., VIZCAINO, P., et al., "Texture imaging of zirconium based components by total neutron cross-section experiments", Journal of Nuclear Materials, v. 425, n. 1-3, pp. 218-227, Jun. 2012.

[5] KAESTNER, A. P., HARTMANN, S., K“UHNE, G., FREI, G., et al., "The ICON beamline - A facility for cold neutron imaging at SINQ", Nuclear Instruments and Methods in Physics Research, v. 659, pp. 387393, Aug 2011.

[6] SANTISTEBAN, J. R., DAYMOND, M. R., JAMES, J. A., et al., "ENGIN-X: a third-generation neutron strain scanner", Applied Crystallography, v. 39, pp. 812-825, Oct 2006.

[7] PIECK, D., "Rediseño de la facultad de neutrografía del RA-6 y su aplicación a la tecnología del hidrogeno", tesis grado, Instituto Balseiro -Universidad de cuyo, San Carlos de Bariloche, 2009.

[8] SIEGMUND, O.H.W., A.S., TREMSIN, VALLERGA, J.V., et al., "Cross-strip anodes for microchannel plate detectors", Space Sciences Laboratory, University of California, Berkeley, CA 94720.

[9] KEARNS, J. J., "Diffusion coefficient of hydrogen in alpha zirconium, Zircaloy-2 and Zircaloy-4" Journal of Nuclear Materials, v. 43, n. 3, pp. 330-338, Jun. 1972. 